\title{
Open Source Library for the Simulation of Wind Power Plants
}

\author{
Philip Eberhart $^{1} \quad$ Tek Shan Chung ${ }^{1} \quad$ Anton Haumer $^{2} \quad$ Christian Kral $^{1}$ \\ ${ }^{1}$ TGM Wien XX, College of Engineering, Austria, dr.christian.kralegmail.com \\ ${ }^{2}$ OTH Regensburg, Germany, anton. haumer@oth-regensburg. de
}

\begin{abstract}
This paper presents the new open source Modelica library WindPowerPlants. For the economic assessment of either a wind power plant or an entire wind park, the accurate prediction of the energy output is essential. Such prediction is usually performed by means of calculations based on statistical wind data. The proposed WindPowerPlants library is capable of assessing the energy output both for statistical and real wind data based on time domain simulations.

In the presented version of the library wind turbine models are modeled with pitch control. The generator models have variable speed and an optional connector to the mains. The entire library is based on power balance conditions and losses are fully neglected. Yet, the library can be extended towards more detailed models considering different types of losses.

The structure and components of the library are presented. Simulations examples are shown and compared with reference data. The applicability of the proposed WindPowerPlants library is demonstrated and possible enhancements are discussed.
\end{abstract}

Keywords: Wind power pants, pitch control, variable speed, energy, power, statistical wind data

\section{Introduction}

Wind power significantly contributes to the total electric energy generation in Europe. Since the economic assessment of future wind power plants is essential, accurate calculations and simulations are needed to predict the energy output of these plants. Therefore, the modeling and simulation of wind power plants is continuously under research. Particular aspects are the wind turbine characteristics (Anderson and Bose (1983); Heier (2009); Ahmed et al. (2014)), the integration of generator models (Mihet-Popa et al. (2004); Yin et al. (2007)), control (Catana et al. (2010); Merabet et al. (2011); Muyeen (2012); Mehdi et al. (2013); Alizadeh and Yazdani (2013)), grid integration (Yuan and Li (2014)) and stability (Du et al. (2014)).

Modelica is particularly suitable to the simulation of wind power plants due to the multi physical domain approach. In Petersson et al. (2012) vertical axis wind power plants and their control aspects are investigated. A paper on variable speed wind turbine models in power system dynamics simulations is published in Enge-Rosenblatt and Schneider (2008). In Strobel et al. (2011) a Modelica library for offshore wind turbines including structural coupling is presented.

The WindPowerPlants library was developed during a Diploma project at the Technical Engineering College, TGM. For the development of the library OpenModelica was used. The library is published under the Modelica license 1.1 and available through GitHub at https://github.com/christiankral/windpowerplants. The main motivations for developing the presented library were:

- Investigate control mechanisms of wind power plants

- Support teaching activities in simulation

- Provide an open source library that may initiate further developments

The proposed library does not model all the controllers that a real wind power plant has. Instead, the intention was to model the overall behavior of wind power plants in such a way, that the major operating conditions are fulfilled. Therefore, only mechanical dynamics are taken into account. Electrical transients are fully neglected. There is yet an electrical interface available to couple electrical networks with one or more wind power plants. This electrical interface to the mains is based on a quasi static multi phase connector.

The WindPowerPlants library is based time domain simulations. Time domain simulations allow a higher flexibility for the investigation of different scenarios than statistical investigations. For example, the impact of time (and temperature) dependent air densities can be investigated. Due to the openness of Modelica, the wind power plant simulation can also be combined with electrical network aspects such as dynamics, stability, etc.

The paper is organized as follows: In Section 2 an overview of the library structure is presented. Section 3 presents the uncontrolled and controlled wind turbine 


\section{WindPowerPlants \\ (i) UsersGuide \\ Experiments \\ Plants \\ . + GenericVariablespeed \\ if GenericVariableSpeedElectrical \\ $\square$ Components \\ -GenericVariableSpeedGenerator \\ -GenericVariableSpeedGeneratorElectrical \\ D IdealRealPower \\ - IdealRealPowerConductance \\ PertchWindTurbine \\ IIT PitchWindTurbineControlled \\ Functions \\ (f) cpVal \\ (..( turbineControlval \\ Blocks \\ : 0 AngularVelocity \\ OAngularVelocityControl \\ $\triangle$ SpeedAdaptor \\ 8.Tipspeed Ratio \\ 出TorqueLimiter \\ $\Delta$ TurbineControlval \\ Records \\ †. $\square$ TurbineControlData \\ †. TurbineData \\ $\square$ WindSources \\ Rayleigh \\ RealData \\ - Sources \\ . Interfaces \\ .. B. BaseWindPowerPlant \\ BaseWindTurbine \\ MSL_322}

Figure 1. Structure of the WindPowerPlants library

models. The variable speed generator models with and without electrical mains connector are explained in Section 4. In Section 5 the structure of the power plants including the control of the generator is shown. Section 6 presents statistical and real wind data sources and Section 7 compares simulation examples with reference data.

\section{Library Structure}

The WindPowerPlants library contains models of entire wind power plants including components. Generic plant models with variable speed generators are located the package Plants, as shown in Fig. 1 .

Package Components contains two models of wind turbines, one with pitch angle input, and another with controlled pitch angle. Until now two generic variable speed generator models are implemented. The first model converts the mechanical power to a power signal connector. The second generator model is equipped with a quasi static three phase connector to the mains and neglects all losses.

In package $\mathrm{Bl}$ locks the controllers, limits, and blocks for the calculation of physical relationships are located. Package Records contains data records of different wind turbines and the optimum pitch angles to maximize the power coefficient and thus power output. WindSources are the data sources of wind velocity signals.

\section{Wind Turbines}

For a given wind speed $v$ the total kinetic power of the wind is

$$
P_{w}=\frac{1}{2} \rho \frac{\pi D^{2}}{4} v^{3}
$$

where $\rho$ is the density of air and $D$ is the rotor diameter. A wind turbine cannot convert the entire kinetic wind power, but only a fraction. This fraction is represented by the power coefficient $c_{p}$. The harvested power is thus determined by the product $c_{p} P_{w}$. The theoretical limit of the power coefficient, $c_{p, \max }=\frac{16}{27} \approx 0.5926$, was determined by Betz; see Heier (2009). The power coefficient of a real wind turbine is always less than this theoretical limit. The actual power coefficient $c_{p}$ relies on the design of the wind turbine and, e.g., the pitch angle. In the proposed wind turbine models it is assumed that the pitch angle (or blade angle) $\beta$ of the blades can be controlled. The pitch angle is the angle of rotation of the blades to control the utilized power. The alternative of stall controlled turbines is not yet included in the WindPowerPlants library. Future wind turbine models may just be modeled by the power coefficient as function of wind speed, since these characteristica are often provided by wind plant manufacturers.

\subsection{Turbine Behavior}

The power coefficient of wind turbines as function of the pitch angle is usually modeled by empiric equations as provided by Heier (2009):

$$
\begin{aligned}
c_{p} & =c_{1}\left(\frac{c_{2}}{\lambda_{1}}-c_{3} \beta-c_{4}\right) e^{-\frac{c_{5}}{\lambda_{1}}}+c_{6} \lambda_{1} \\
\lambda_{1} & =\frac{1}{\frac{1}{\lambda+0.089}-\frac{0.035}{\beta^{3}+1}}
\end{aligned}
$$

In these equations $c_{1}, \ldots, c_{6}$ are turbine specific per unit coefficients and $\lambda_{1}$ is the internal wind tip ratio which in turn relies on the tip speed ratio

$$
\lambda=\frac{\omega D}{2 v}
$$


Table 1. Wind turbine coefficients of different literature references

\begin{tabular}{lcc} 
& Heier (2009) & Thongam et al. (2009) \\
\hline$c_{1}$ & 0.5 & 0.5176 \\
$c_{2}$ & 116.0 & 116.0 \\
$c_{3}$ & 0.4 & 0.4 \\
$c_{4}$ & 5.0 & 5.0 \\
$c_{5}$ & 21.0 & 21.0 \\
$c_{6}$ & 0.0 & 0.006795 \\
\hline
\end{tabular}

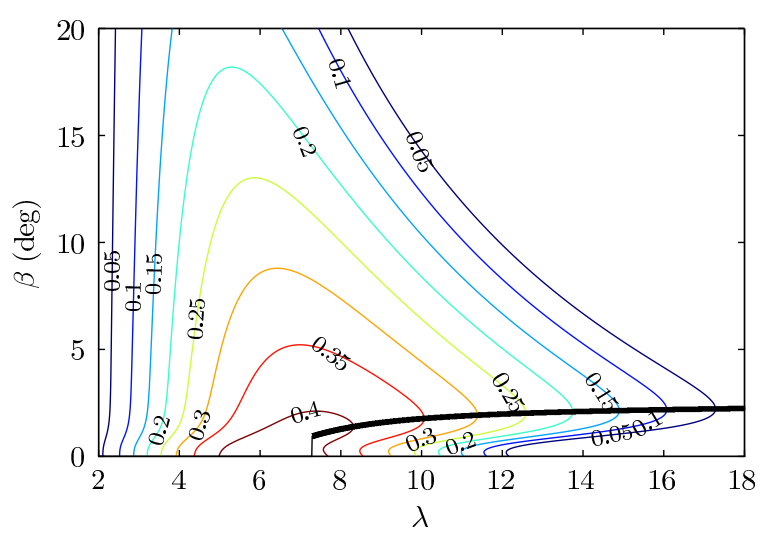

Figure 2. Curves of constant power coefficients, $c_{p}$, as a function of the tip speed ratio, $\lambda$, and the pitch angle $\beta$ for the wind turbine coefficients given by Heier (2009)

and the pitch angle $\beta$ in degrees. The tip speed ratio is the ratio of the peripheral speed over wind speed. The peripheral speed equals the angular velocity $\omega$ times half the diameter.

The wind turbine coefficients $c_{1}, \ldots, c_{6}$ also reflect the actual geometry of the blades. Examples of turbines from Heier (2009) and Thongam et al. (2009) are compared in Tab. 1. For the wind turbine coefficients given by Heier (2009) curves of constant power coefficients are depicted in Fig. 2. The bold black curve in this figure indicates the curve of maximum $c_{p}$ for variable tip speed ratio. This curve represents the optimum pitch angle as function of tip speed ratio. The bold black curve is approximated by a third order polynomial function

$$
\beta_{\mathrm{opt}}=p_{1} \lambda^{3}+p_{2} \lambda^{2}+p_{3} \lambda+p_{4}
$$

The polynomial coefficients $p_{1}, \ldots, p_{4}$ are stored in a parameter record matching the turbine model and have to be determined by a pre-processing tool. From Fig. 2 it is also obvious that there is the region for $\lambda<7.3$ where $\beta=0$ leads to the optimum power coefficient. For $\lambda>18.5$ the pitch angle $\beta=0$ leads to the optimum power coefficient; this region is, however, not depicted in Fig. 2.

The approximation of the power coefficient in (2) and (3) has the drawback that negative pitch angles cause non-meaningful results - even though negative pitch angles are used in practice. However, the actual wind power shall be limited by positive values to avoid plausibility problems,

$$
P=\max \left(0, c_{p} P_{w}\right)
$$

\subsection{Uncontrolled Wind Turbine}

The signal inputs of the uncontrolled wind turbine model are the wind velocity $v$ and the pitch angle $\beta$. The turbine model also has a rotational connector taken from Modelica. Mechanics. Rotational. Interfaces. When the wind velocity exceeds a certain threshold, the turbine reaches the power limiting range. In this case the boolean output limit becomes true. Additional signal outputs are the tip speed ratio, $\lambda$, and the angular velocity, $\omega$.

The uncontrolled wind turbine model is coded textually. The most relevant equations are:

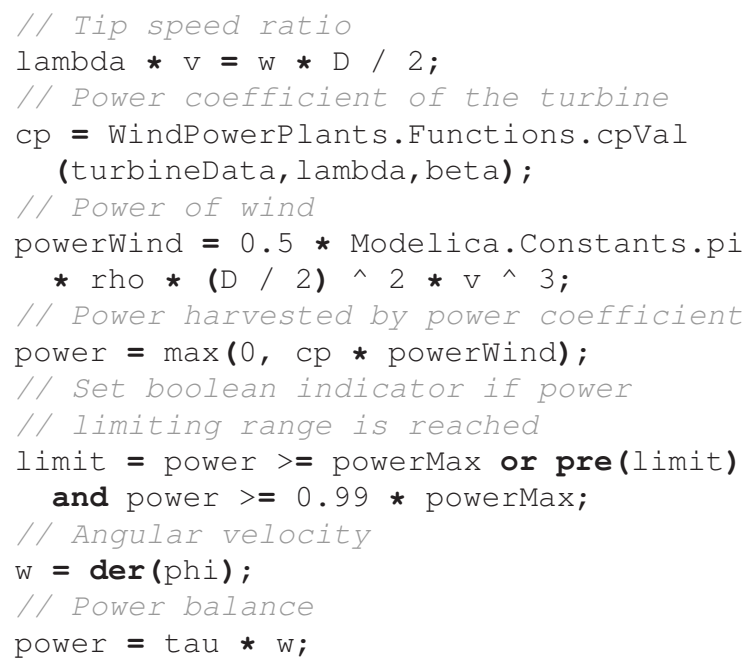

In order to operate the uncontrolled wind turbine model, signal inputs for the wind velocity, $v$, and the pitch angle, $\beta$, are required. In a simulation model of a full wind power plant the pitch angle shall be provided by a controller. Based on the uncontrolled wind turbine model a standard controlled wind turbine model is included in the library.

\subsection{Controlled Wind Turbine}

The standard controlled wind turbine model provided in the PowerPlantsLibrary and shown in Fig. 3 utilizes the uncontrolled turbine model of Subsection 3.2. In this model the pitch angle $\beta$ is controlled for two different operating ranges of the wind turbine. The two different ranges are indicated by the boolean output limit of the uncontrolled wind turbine model:

- optimum power coefficient range: limit $=$
false

- power limiting range: limit = true 


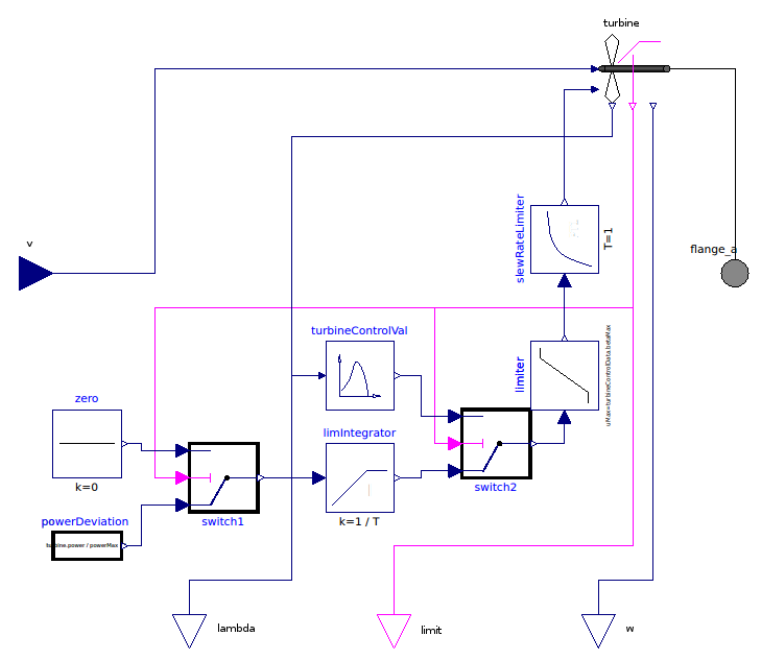

Figure 3. Controlled wind turbine

In the optimum power coefficient range the pitch angle $\beta$ is determined by the polynomial approximation described in Subsection 3.1. This task is accomplished by the block turbineControlval depicted in Fig. 3. The actual pitch angle is then limited by the interval $\left[0^{\circ}, 90^{\circ}\right]$. After limiting $\beta$, a first order delay is used to smoothen the pitch angle response. In a real wind power plant the pitch angle slope is limited. For simplicity reasons, a first oder delay is used in the WindPowerPlants library.

When the wind turbine is in the power limiting range, the pitch angle has to be controlled such way that the actual power $P$ does not exceed the maximum power $P_{\max }$. The maximum power $P_{\max }$ is a parameter of the wind turbine model. In the power limiting range the relative power deviation

$$
\Delta p=\frac{P}{P_{\max }}-1
$$

is controlled to be zero. For this purpose a fast limiting integral controller is used which processes (7).

\section{Variable Speed Generators}

The implemented variable speed generators are generic. This means, that typical electrical characteristics of either induction or synchronous generator are not taken into account. The generator models solely rely on power balances and no losses are taken into account.

In the actual implementation the maximum angular velocity of the generator is not limited. However, a real generator does have a maximum angular velocity and frequency, respectively. The difference between the real and the modeled behavior are

- different tip speed ratios,

- different power coefficients,

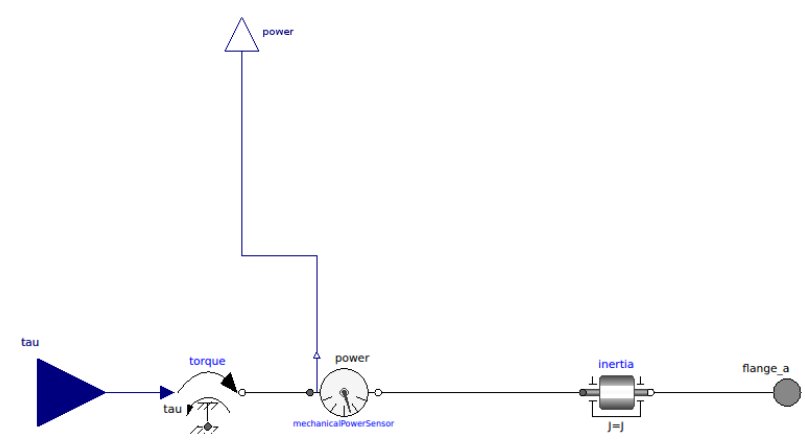

Figure 4. Generic variable speed generator model

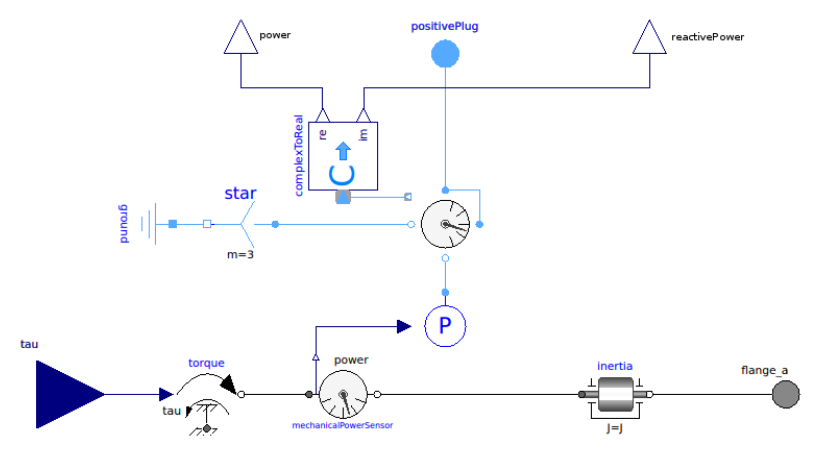

Figure 5. Generic variable speed generator model with electrical connector to the mains

but the output power is the same, since the maximum angular velocity occurs in the power limiting range. Therefore, there is no difference in the total energy output.

Each of the two variable speed generator models shown in Fig. 4 and 5 have one signal input connector: the reference torque. The reference torque is determined by the torque controller of the power plant. In the generic generator models the torque signals are converted into a physical connector quantities. This means for each model, that the reference torque and the actual torque are always equal. A power sensor is used to provide the power output connector with the actual mechanical power. The total inertia of the generator is considered.

In the generator model with electrical connector to the mains (Fig. 5) a power balance model is utilized using a quasi static multi phase connector from Modelica.Electrical.Quasistationary. Multiphase. Interfaces. In the current implementation it is assumed that a grid inverter controls the reactive power $Q$ to be zero. The output of the power sensor is used to operate an ideal electrical multi phase power source which generates real power. In the WindPowerPlants library two ideal power sources are provided. The power source WindPowerPlants. Components. IdealRealPower used in Fig. 5 utilizes the voltage and current space phasor Haumer et al. (2008). The current space phasor is controlled in such a way that the power input signal of the source and the 


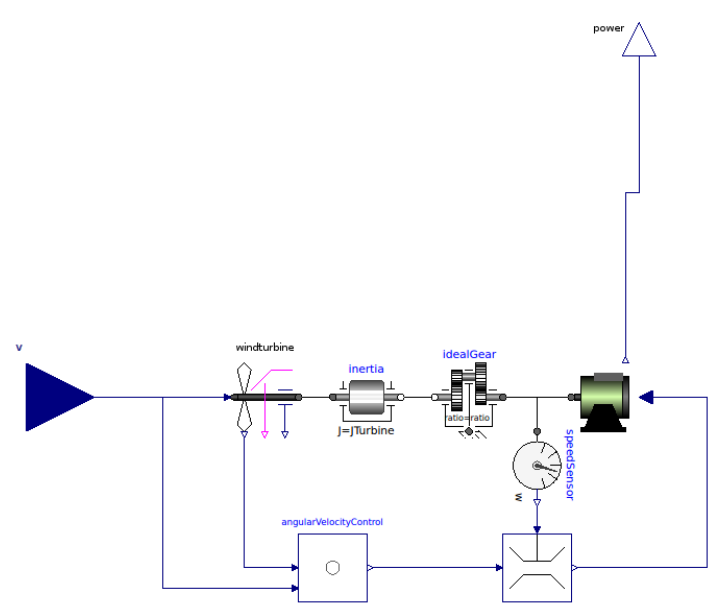

Figure 6. Model of a generic power plant

total real power of all phases are equal. This requires the current space phasor to be aligned with voltage space phasor in order to achieve zero reactive power. In the alternative power source WindPowerPlants. Components. IdealRealPower Impedance shown in Fig. 1 the real power is controlled by means of a multi phase conductor to achieve $100 \%$ power balance between the signal input and the three phase electrical power. However, the limitation of $Q=0$ can be overcome in the future by also controlling the reactive power. By controlling the reactive power the voltage of the wind power plant can be controlled.

\section{Wind Power Plants}

\subsection{Model Structure}

The structure of the generic power plant models with and without electrical connector to the mains are depicted in Fig. 6 and 7, respectively. The reason why both models are named generic, is that they are based on a generic variable speed generator.

The input connector of the power plant model is the wind speed. The wind speed is connected with the wind turbine model which converts the fraction $c_{p}$ of the kinetic wind power $P_{w}$ into mechanical power $P$ according to (6). The wind turbine model is connected with an inertia, representing the rotating inertias of the wind turbine and the gear with respect to the wind turbine rotational angular velocity. The ideal gear represents the multi stage planetary gear of the real wind power plant. The generator is directly coupled to the high speed side of the gear. The torque input of the generator is controlled by the block angularVelocitycontrol. The torque control is then limited by a rotor speed dependent torque limiter in order to avoid negative rotor speed and high torque dynamics around zero speed.

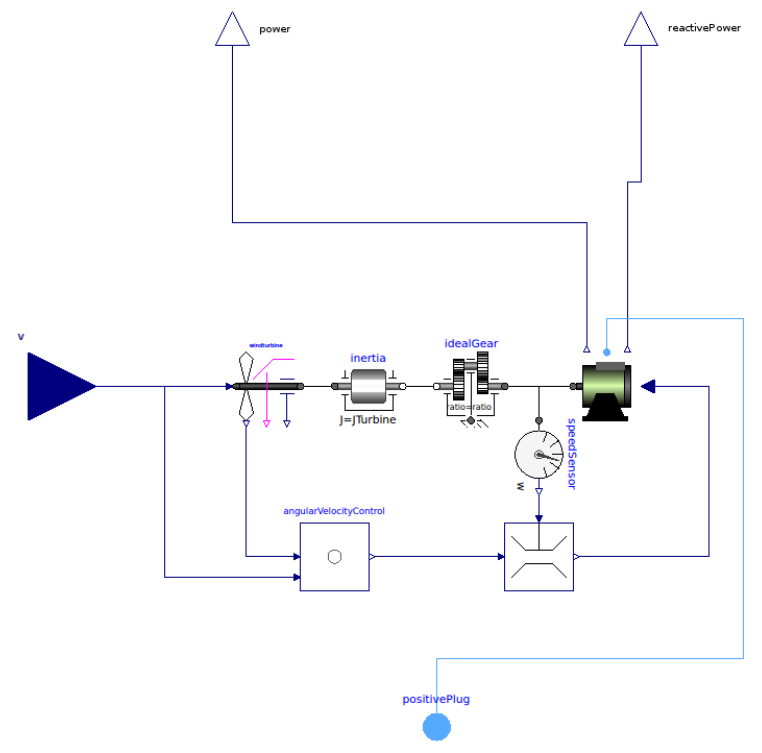

Figure 7. Model of a generic power plant with electrical mains connector

\subsection{Angular Velocity Control}

In the angular velocity control of the generator two different wind speed regions are distinguished. In the stand still region, for wind speeds $v<v_{\min }$, the wind turbine is controlled towards zero angular velocity $\omega_{\text {ref }}$ and thus zero reference tip speed ratio, $\lambda_{\text {ref }}$. The wind speed $v_{\text {min }}$ represents the cut-in speed of the turbine which is in the range of approximately $4 \mathrm{~m} / \mathrm{s}$. In the power generating region, for $v \geq v_{\min }$, regular power conversion occurs. The cut-out speed is currently not considered in the control.

In the implemented control strategy the reference value of the tip speed ratio, $\lambda_{\text {ref }}$, is set depending on the wind speed region. For the stand still region $\lambda_{\text {ref }}=0$ is chosen. For the power generating region the reference tip speed ratio is derived from the optimum power coefficient as described in Section 3. The optimum power coefficient is the maximum $c_{p}$ as a function of the actual tip speed ratio; see the bold black curve in Fig. 2. The maximum power coefficient is indicated by two variables, $\lambda_{\text {opt }}$ and $\beta_{\text {opt }}$. However, as the variable $\lambda_{\text {opt }}$ represents the optimum tip speed ratio, the optimum pitch angle, $\beta_{\text {opt }}$, is automatically determined from the polynomial approximation of maximum $c_{p}$ values indicated by (5). This way the control always achieves the highest power coefficient in the optimum power coefficient range.

In Fig. 8 the reference tip speed ratio, $\lambda_{\text {ref }}$, is the output of block switch1. From the reference and actual tip speed ratio the deviation of the reference and actual tip speed ratio

$$
\Delta \lambda=\lambda_{\text {ref }}-\lambda
$$

is calculated. This deviation corresponds with the angu- 


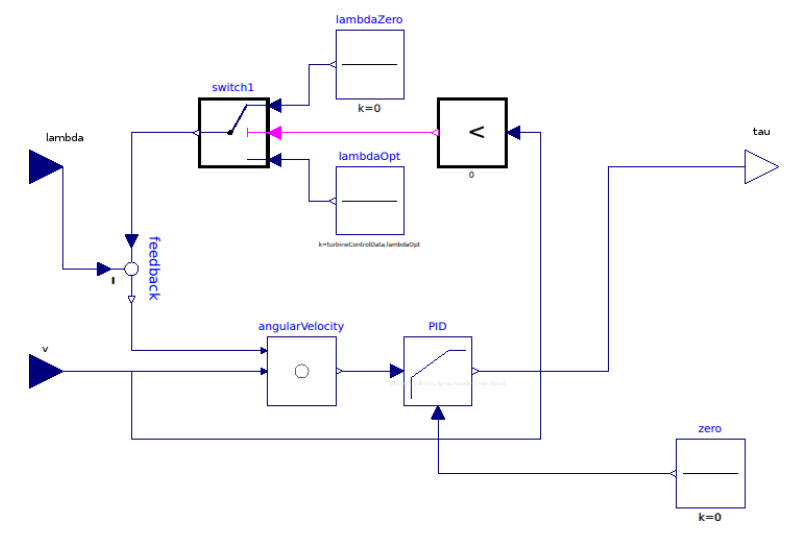

Figure 8. Angular velocity control of wind turbine

lar speed deviation

$$
\Delta \omega=\frac{2 v \Delta \lambda}{D}
$$

according to (4). The calculation of (9) is achieved by the block angularVelocity in Fig. 8. The angular speed deviation $\Delta \omega$ is fed to the reference input of a PI controller. As the used PI controller has both a reference and a sensor input, the sensor input set to zero, as the difference of these reference and sensor signal are already determined by (9). The output of the PI controller is the reference torque of the generator.

\subsection{Torque Limitation}

The torque output of the angular velocity control is limited to avoid significant dynamics around zero speed and for negative speeds. Since the electric machine is operated as generator, the mechanical power

$$
P_{m}=\tau \omega
$$

is negative. The generator is operated at positive angular speed $\omega$ and thus torque $\tau$ is negative. Torque limitation, however, is implemented as function of speed. The characteristic of the torque limiter is shown in Fig. 9 for the input torque $\tau=-10 \mathrm{kNm}$ and a torque limit of $\tau_{\text {ref }}=10 \mathrm{kNm}$. However, In the investigated case the reference speed $\omega_{\text {ref }}=50 \pi \mathrm{rad} \mathrm{s}^{-1}$. For positive speeds greater than $2 \%$ of the reference speed the torque is not limited. The range between zero angular speed and $2 \%$ of the reference speed torque is limited by the steep linear curve shown in Fig. 9. In the negative speed range which shall never be reached - torque is limited linearly towards the negative reference angular velocity.

\section{Wind Sources}

In the WindPowerPlants library two different wind sources are provided.

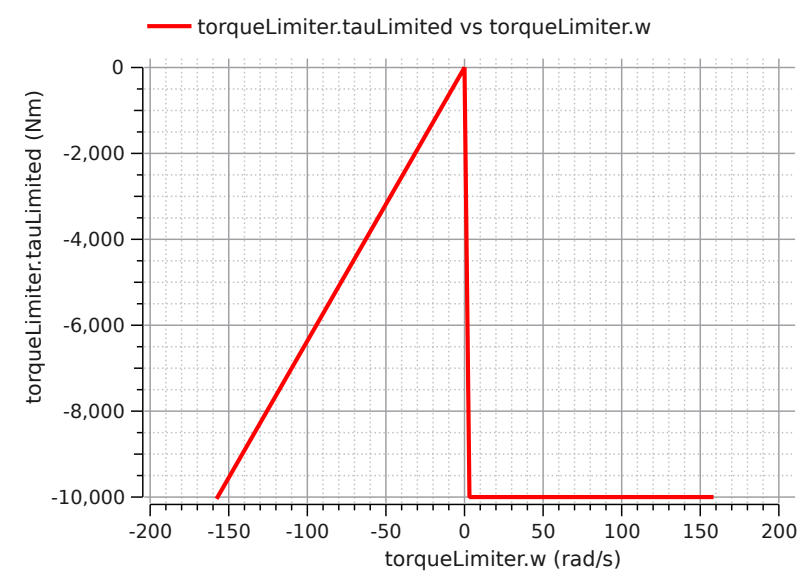

Figure 9. Characteristic of the torque limiter

\subsection{Real Wind Data}

The first wind data source reads a data file with at least two columns. The first column represents time (in seconds) and the second column is the actual wind velocity (in $\mathrm{m} / \mathrm{s}$ ). The real data wind source model is directly derived by a CombiTimeTable, limited to on single parameter: the file name.

\subsection{Statistical Wind Data}

The second wind source provides the wind speed as function of time, calculated by the discrete Rayleigh distribution

$$
d_{k}=\frac{\pi}{2} \frac{k \Delta v^{2}}{v_{m}^{2}} \exp \left(-\frac{\pi}{4} \cdot \frac{k^{2} \Delta v^{2}}{v_{m}^{2}}\right) .
$$

In this equation $k$ is the interval index, considering $n$ intervals in total. The speed interval

$$
\Delta v=\frac{v_{\max }}{n}
$$

is derived from the maximum speed and $v_{m}$ represents the average value of all wind speeds. The variables $d_{k}$ represent the relative duration of each wind interval with index $k$. The sum of all distributions is equal to one,

$$
\sum_{k=1}^{n} d_{k}=1
$$

For a time domain representation the total time period $T$ is utilized. In a sequence of $n$ different wind speeds

$$
v_{k}=k \cdot \Delta v
$$

the duration of the respective wind speed is the determined by $d_{k} T$. An example of a histogram of Rayleigh distributed wind data is shown in Fig. 10. The respective wind speeds versus time are depicted in Fig. 11. For a particular index $k$ and the accessory wind speed (14) the distribution $d_{k}$ determines the duration for which the wind speed is constant. After this duration the next wind 


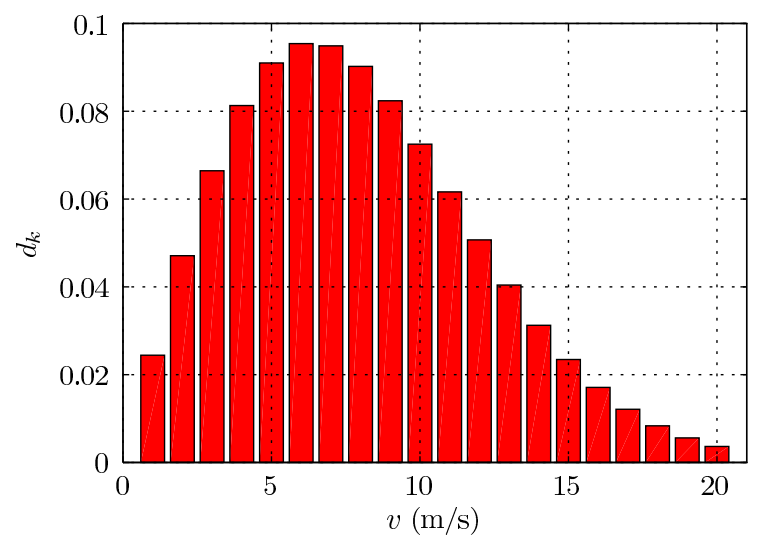

Figure 10. Histogram of Rayleigh distributed wind speed for $v_{\max }=20 \mathrm{~m} / \mathrm{s}, v_{m}=7 \mathrm{~m} / \mathrm{s}$ and $n=20$

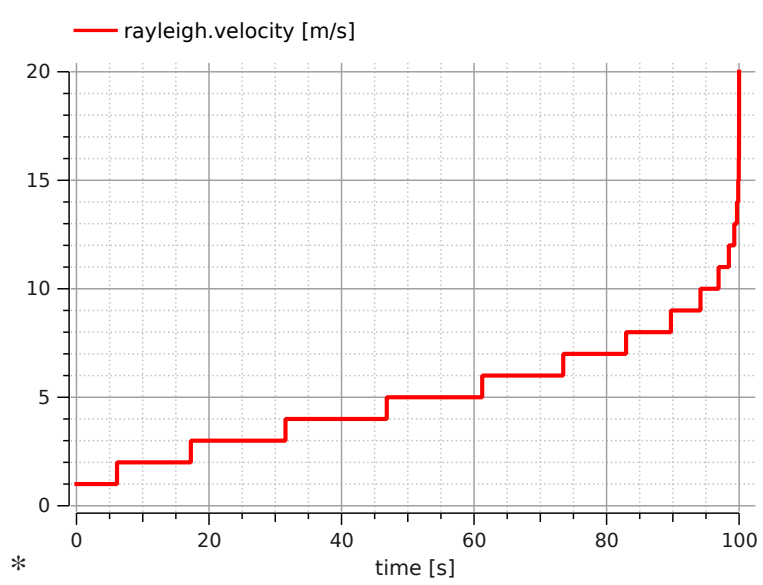

Figure 11. Rayleigh distributed wind speeds in the time domain for $v_{\max }=20 \mathrm{~m} / \mathrm{s}, v_{m}=7 \mathrm{~m} / \mathrm{s}, n=20$ and a period $T=100 \mathrm{~s}$

speed index is processed up to index $n$. This procedure may be continued periodically. Due to the characteristic of the Rayleigh distribution higher wind speeds and indexes, respectively, give rise for a lower distribution and thus for shorter duration of a constant speed. This is why the curve shows the steepest rise towards the end of the period $T$.

\section{Application Examples}

In order to validate the developed wind power plant models, reference calculation data of planned wind power plants are compared with simulation results. An example of a simulation model is depicted in Fig. 6. The reference calculation data used in this paper are provided from the authorities of the province of Lower Austria. Reference data are available from two different wind farms. From the two wind farms altogether three reference power plants are selected. The two wind farms are not explicitly named in this paper to protect wind farm operator data. Instead name synonyms are used. The reference power data were calculated by the appli-

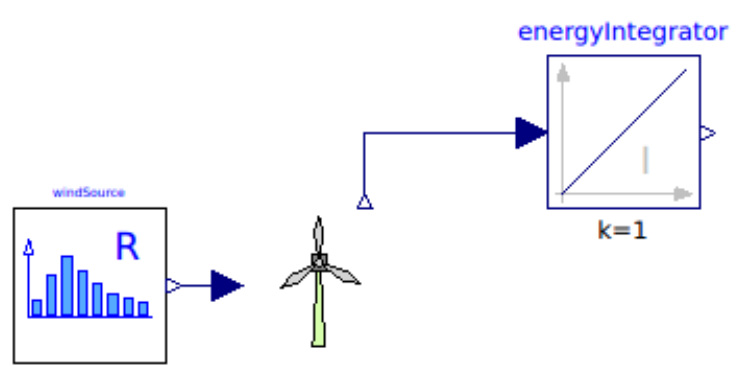

Figure 12. Simulation experiment with statistical wind data

Table 2. General and technical data of the reference wind power plants

\begin{tabular}{rcccc}
\hline & \multicolumn{5}{c}{ case } \\
\hline Wind farm & unit & A & B & C \\
Plant number & & 1 & 2 & 15 \\
Manufacturer Vestas - type & & V112 & V112 & V90 \\
Power rating & MW & 3 & 3 & 2 \\
Gear ratio & & 112.8 & 112.8 & 112.8 \\
Rotor diameter & $\mathrm{m}$ & 112 & 112 & 90 \\
Height of hub & $\mathrm{m}$ & 135 & 135 & 125 \\
\hline
\end{tabular}

cants of the wind farms using the software WAsP (Wind Atlas Analysis and Application Program). The general and technical data of the investigated wind power plants and are summarized in Tab. 2. The simulations are performed in OpenModelica on a notebook computer with Intel Core ${ }^{\mathrm{TM}} 2$ Duo CPU T7250 operated at $2 \mathrm{GHz}$ and $2 \mathrm{~GB}$ of main memory.

A comparison of the power plant data, and the reference and simulation data is presented in Tab. 3. The annual energy harvest is indicated as total Energy in MWh divided by one year (1a), i.e., the physical quantity of power. The deviations of the simulation results are in the range of $\pm 7 \%$. The main causes of these deviation are:

- The exact characteristic of the power coefficient of

Table 3. Variables and calculation data of reference (ref) and simulation (sim) wind power plants

\begin{tabular}{rcccc} 
& & \multicolumn{3}{c}{ case } \\
\hline quantity & unit & A & B & C \\
\hline air density $\rho$ & $\mathrm{kg} / \mathrm{m}^{3}$ & 1.198 & 1.198 & 1.198 \\
average wind speed $v_{m}$ & $\mathrm{~m} / \mathrm{s}$ & 6.95 & 7.12 & 7.30 \\
maximum wind speed $v_{\max }$ & $\mathrm{m} / \mathrm{s}$ & 25 & 25 & 25 \\
number of speed intervals $n$ & & 25 & 25 & 25 \\
interval of investigation & $\mathrm{h}$ & 8760 & 8760 & 8760 \\
sampling interval & $\mathrm{s}$ & 10 & 10 & 10 \\
CPU time & $\mathrm{s}$ & 104 & 104 & 104 \\
\hline power (reference) & $\mathrm{MWh} / \mathrm{a}$ & 10122 & 10636 & 6488 \\
power (simulation) & $\mathrm{MWh} / \mathrm{a}$ & 9497 & 9905 & 6869 \\
absolute power deviation & $\mathrm{MWh} / \mathrm{a}$ & -625 & -731 & +381 \\
relative power deviation & $\%$ & -6.2 & $-6,8$ & +5.9 \\
\hline
\end{tabular}


the wind turbine is not known; all simulations results are achieved using the wind turbine parameters of Heier (2009)

- The reference data are based on real measured wind speed data whereas the simulations rely on a Rayleigh distribution of the wind speed

Considering on what information is available on the reference power pants, the quantitative simulation results are sufficiently accurate.

\section{Conclusion}

A new open source Modelica library for the simulation of wind power plants is presented. The library structure and the main components are explained. The control strategies of the pitch angle and the angular velocity of the wind turbine are described. Three reference wind power plants are compared with simulation results. The average power deviations of the simulations from the reference data are in the range of $\pm 7 \%$.

\section{References}

S. Ahmed, M.A Rashid, S.B. Yaakob, and A Yusof. Pitch angle control of a grid connected wind turbine. Intelligent and Advanced Systems (ICIAS), 2014 5th International Conference on, pages 1-6, June 2014. doi: 10.1109/ICIAS.2014. 6869523.

O. Alizadeh and A. Yazdani. A strategy for real power control in a direct-drive pmsg-based wind energy conversion system. Power Delivery, IEEE Transactions on, 28(3):12971305, July 2013. ISSN 0885-8977. doi: 10.1109/TPWRD. 2013.2258177.

P.M. Anderson and Anjan Bose. Stability simulation of wind turbine systems. IEEE Transactions on Power Apparatus and Systems, PAS-102(12):3791 - 3795, 12 1983. ISSN 0018-9510. doi: 10.1109/TPAS.1983.317873.

I. Catana, C. A. Safta, and V. Panduru. Power optimization control system of wind turbines by changing the pitch angle. U.P.B. Sci. Bull., Series D, 72(1):141-148, 2010.

Zhaobin Du, Jingshang Chen, Yaopeng Huang, Peng Shen, and Shangyun Liu. The research of simplification of doubly-fed wind turbine in the small signal stability analysis. Power and Energy Engineering Conference (APPEEC), 2014 IEEE PES Asia-Pacific, pages 1-6, Dec 2014. doi: 10.1109/APPEEC.2014.7066161.

Olaf Enge-Rosenblatt and Peter Schneider. Modelica wind turbine models with structural changes related to different operating modes. Modelica Conference, 2008.
A. Haumer, C. Kral, J. V. Gragger, and H. Kapeller. Quasistationary modeling and simulation of electrical circuits using complex phasors. International Modelica Conference, 6th, Bielefeld, Germany, pages 229-236, 2008.

Siegfried Heier. Windkraftanlagen: Systemauslegung, Netzintegration und Regelung. Vieweg + Teubner, 5 edition, 2009.

Mehdi, Allagui1, Othman B.k, Hasnaoui, Jamel, and Belhadj. Exploitation of pitch control to improve the integration of a direct drive wind turbine to the grid. J. Electrical Systems, 9(2):179-190, 2013.

A Merabet, J. Thongam, and J. Gu. Torque and pitch angle control for variable speed wind turbines in all operating regimes. Environment and Electrical Engineering (EEEIC), 2011 10th International Conference on, pages 1-5, May 2011. doi: 10.1109/EEEIC.2011.5874598.

L. Mihet-Popa, F. Blaabjerg, and I Boldea. Wind turbine generator modeling and simulation where rotational speed is the controlled variable. Industry Applications, IEEE Transactions on, 40(1):3-10, Jan 2004. ISSN 0093-9994. doi: 10.1109/TIA.2003.821810. Wind turbine control pitch power torque.

S.M. Muyeen. Wind Energy Conversion Systems. Springer, 2012. ISBN 978-1-4471-2201-2.

Joel Petersson, Hubertus Tummescheit, Pär Isaksson, and Johan Ylikiiskilä. Modeling and simulation of a vertical wind power plant in dymola/modelica. Proceedings of the 9 th International Modelica Conference, 2012. doi: 10.3384/ecp12076631.

M. Strobel, F. Vorpahl, C. Hillmann, X. Gu, A. Zuga, and U. Wihlfahrt. The onwind modelica library for offshore wind turbines - implementation and first results. Modelica Conference, 2011.

J.S. Thongam, P. Bouchard, H. Ezzaidi, and M. Ouhrouche. Wind speed sensorless maximum power point tracking control of variable speed wind energy conversion systems. Electric Machines and Drives Conference, 2009. IEMDC '09. IEEE International, pages 1832-1837, May 2009. doi: 10.1109/IEMDC.2009.5075452.

Ming Yin, Gengyin Li, Ming Zhou, and Chengyong Zhao. Modeling of the wind turbine with a permanent magnet synchronous generator for integration. Power Engineering Society General Meeting, 2007. IEEE, pages 1-6, June 2007. ISSN 1932-5517. doi: 10.1109/PES.2007.385982.

Xibo Yuan and Yongdong Li. Control of variable pitch and variable speed direct-drive wind turbines in weak grid systems with active power balance. Renewable Power Generation, IET, 8(2):119-131, March 2014. ISSN 1752-1416. doi: 10.1049/iet-rpg.2012.0212. 